\title{
Best Practices and Methodologies for IT Project Portfolio Management
}

\section{Raúl H. Rosero¹, Igor Aguilar Alonso², Maria A. Procel³, and Omar S. Gómez}

${ }^{1}$ Grupo de Investigación en Ingeniería de Software (GRIISOFT), Facultad de Informática y Electrónica, Escuela Superior Politécnica de Chimborazo, Riobamba - Ecuador

${ }^{2}$ Facultad de Informática, Universidad Politécnica de Madrid, Madrid - España

3Unidad de Admisión y Nivelación, Escuela Superior Politécnica de Chimborazo, Riobamba Ecuador

\section{Abstract}

In today's business environment, a key factor for decision making is the IT projects portfolio management (ITPPM). IT projects must be aligned with the objectives, goals and strategies, which is achieved through the appropriate application of a methodology for ITPPM. The experienced application of a methodology depends

Corresponding Author:

Raúl H. Rosero

rrosero@espoch.edu.ec

Received: 28 July 2017

Accepted: 5 September 2017

Published: 30 January 2018

Publishing services provided by

Knowledge $\mathrm{E}$

(c) Raúl H. Rosero et al. This article is distributed under the

terms of the Creative Commons

Attribution License, which

permits unrestricted use and

redistribution provided that the

original author and source are credited.

Selection and Peer-review under the responsibility of the SIIPRIN Conference Committee.

\section{G OPEN ACCESS}

on the experience of the managers of ITPPM, considering that the application of methodologies generates best practices, it becomes necessary to study some of the more representative methodologies and obtain the best practices that allow novice administrators to initiate their portfolio management activities in information technology projects. In this article, three methodologies are studied, from which best practices are obtained, as results; a hierarchy of best practices is obtained so that project managers can be guided or introduced in their field.

Keywords: IT Project Portfolio Management, Business Solutions, IT Strategic Demand Management, IT Project Portfolio Management Methodologies, IT Project Portfolio Management Best Practices

\section{Introducción}

La gestión de portafolio de proyectos de tecnologías de la información (GPPTI) se ha convertido en los últimos tiempos en uno de los puntales principales del desarrollo de la empresas, que permite que la planificación y despliegue de proyectos de tecnología estén alineados con las metas y estrategias de las empresas, lo cual implica retos como la especificación de una estructura apropiada para la definición, la implementación y evaluación de proyectos de tecnología de TI, un soporte tecnológico para la consecución de objetivos estratégicos y finalmente establecer un apropiado control de cambios en los proyectos principalmente cuando las estrategias empresariales se modifican [1]. 
Según los estudios de Aguilar Alonso [2], indican que la demanda de Tl es un proceso clave para lograr el éxito del gobierno de la TI, la demanda de la TI es muy amplia y variada que proviene de diferentes direcciones y en diferentes formas, tanto del interior de la empresa, así como del exterior, por lo que la demanda de TI es segmentada en tres grandes categorías: 1) Demanda estratégica, 2) Demanda táctica y 3) Demanda operacional [3], cada una de estas demandas son asignadas a diferentes portafolios de TI. La demanda estratégica de la TI, es asignada al portafolio de proyectos de TI para su gestión. La gestión del portafolio de proyectos de TI (GPPTI) ofrece un proceso basado en eventos que pueda consolidar, evaluar, priorizar y monitorear los proyectos de $\mathrm{TI}$ [4], éstas a su vez generan métricas "post-mortem" de los proyectos que ayuden a mejorar las buenas prácticas en la organización.

Lo anterior se consigue a través de la aplicación apropiada de metodologías de gestión del portafolio de proyectos de TI, para tomar decisiones oportunas que garanticen que los beneficios ofrecidos sean igualmente oportunos [1].

La literatura evidencia que la aplicación de metodologías de GPPTI está basado en reportes anecdóticos [5], siendo por lo tanto subjetiva la aplicación de sus resultados.

En este artículo se analizan varias metodologías y sus prácticas, a partir de ellas establecer una priorización de mejores prácticas, de tal manera que posibilite a que los administradores de una oficina de gestión de proyectos de TI puedan guiarse o introducirse en su actividad.

El objetivo del trabajo es recopilar información representativa y detallada que sirva como referencia para seleccionar la(s) metodología(s) y las mejores prácticas para la gestión del portafolio de proyectos de TI.

Este artículo está organizado como sigue: en la sección 2 se presenta un marco de referencia de la Teoría Moderna de Proyectos (TMP), la Gestión de Proyectos y la Gestión de proyectos de TI. En la sección 3 se presenta la metodología de trabajo aplicada en el artículo. Los resultados y su discusión sobre las metodologías y mejores prácticas determinadas en las secciones 4 y 5 . Finalmente la sección 6 presenta las conclusiones.

\section{Antecedentes}

A continuación se presenta un conjunto de definiciones y conceptos en el marco de la gestión de portafolio de proyectos de TI conjuntamente con su evolución en el tiempo que sirven como marco referencial para el resto del documento. 


\subsection{Teoria Moderna de Portafolio (TMP)}

En 1938 Jhon Burn Williams, en su libro "Teoría del Valor de la Inversión" [6] menciona que las inversiones financieras se realizaban en base al análisis del retorno de las mismas. Más tarde Harry Markowits (Premio Nobel 1990) publica en 1952 un artículo en el "Journal of Finance" titulado "Portfolios Selection" [7], en el que introduce el factor riesgo y su análisis como parámetro para la selección de inversiones, con lo que cambia radicalmente la forma como los inversionistas toman decisiones con respecto a sus proyectos de inversión.

Lo anterior da inicio a la Teoría Moderna de Portafolios (TMP), que resumidamente establece que para un determinado nivel de riesgo, existe una combinación especifica de inversiones que alcanzara un retorno optimo [8], adicionalmente, menciona que las inversiones no se deben seleccionar individualmente basándose en las características de riesgo/retorno de cada una, sino que los inversionistas deben seleccionar sus portafolios en base a las características globales del factor riesgo/retorno del portafolio de acuerdo a dos principios básicos: maximizar el retorno para un determinado riesgo o minimizar el riesgo para un determinado retorno.

Teniendo como base la inversión financiera, actualmente se está aplicando a la gestión de proyectos de tecnología de la información (TI), combinando la GPPTI y la gestión de portafolios de inversión, dado que persigue las mismas metas. Cabe resaltar que la teoría de Markowits no se puede aplicar directamente al GPPTI, porque al momento de iniciar un proyecto de TI no se puede recuperar la inversión hasta que finalice el proyecto, lo que no sucede, por ejemplo, con los bonos y acciones.

\subsection{TMP aplicado a proyectos de TI}

Como se mencionó anteriormente es factible aplicar la Teoría Moderna de Portafolios para la gestión de portafolios de proyectos de $\mathrm{TI}$, siempre que se considere algunas restricciones que tiene que ver con el riesgo inherente a los proyectos de TI [1]. Entre los más sobresalientes podemos mencionar a los siguientes:

- Los riesgos del mercado: tienen que ver con los cambios inesperados con la demanda del mercado lo que provoca que los ejecutivos cambien las estrategias del negocio.

- Los riesgos organizacionales: tienen que ver con la apertura empresarial hacia una solución de $\mathrm{TI}$, lo que también tiene que considerar el nivel de madurez empresarial 
- Los riesgos técnicos: que tienen que ver con alguna característica para cumplir una funcionalidad, diseño, implementación, ambigüedad, u obsolescencia de TI.

- Los riesgos de procesos o proyectos: tienen que ver con los riesgos de cumplir un determinado cronograma o presupuesto, también se considera lo que tiene que ver con los riesgos de identificar los requerimientos del proyecto, al de la gestión de capital humano.

Markowits demostró que los portafolios de inversiones con un alto riesgo tiene a su vez un alto retorno a la inversión [8], esta situación no se presenta en proyectos de TI, ya que la medición del riesgo y retorno es más complejo, por ejemplo en el ámbito financiero se trabajó con análisis de tendencias mientras que en proyectos de TI se maneja escenarios o hechos [9].

Por lo que se recomienda que estos factores sean manejados a través de una oficina de administración de portafolios de proyectos de TI (OGP).

\subsection{Diferencias entre la gestión de proyectos y la gestión de portafolio de proyectos}

Los dos términos a menudo se los considera similares, sin embargo son muy diferentes según el Project Management Institute (PMI) [10], la gestión de proyectos (GP) es un proceso que se enfoca básicamente en que se logre los objetivos en función de sus costos, calendarios técnicas y rendimientos, la gestión de proyectos tiene un inicio y un fin definido, en cambio la gestión de portafolio de proyectos (GPP) es una actividad más amplia y constante dentro de la organización y su principal objetivo es identificar, seleccionar, financiar, monitorear y mantener una combinación adecuada de proyectos e iniciativas necesarias para alcanzar las metas de la organización [11]

Así, La GPP considera la suma de costos, riesgos rendimientos de todos los proyectos dentro del portafolio, así como los diversos intercambios entre ellos, sin descuidar el estado individual de cada proyecto dentro del portafolio.

Con respecto a las actividades principales de cada una tenemos que la GP se encarga de preparar el cronograma del proyecto, preparar presupuesto, autorizar gastos, mantener comunicación con los auspiciantes del proyecto, dirigir al equipo del proyecto entre las principales; en cambio la GPP es más amplia y se encarga de la gestión de varios proyectos sea preparando planes a largo plazo para la toma de decisiones apropiadas sobre la asignación de recursos, supervisar que los proyectos se ejecuten a tiempo y dentro del presupuesto, asignar personal para la gestión de proyectos entre otras. 
Maizlishy Handler [12] en su libro "IT Portfolio Management step by step" establece que para que la TI ofrezca valor agregado a la empresa, debe considerar los siguientes aspectos:

- priorización y alineación con la visión corporativa,

- inversiones balanceadas en todas las unidades de la empresa,

- mecanismo pragmático de costos y control de riesgos,

- procesos racionales de toma de decisiones,

- flexibilidad para revalorar y re balancear prioridades,

- cumplimiento de requerimientos regulatorios y legales.

\subsection{Gestión de Portafolio de Proyectos y la Oficina de Gestión de Portafolio}

A fin de minimizar los inconvenientes y desventajas que implica la gestión no integrada de proyectos se propone iniciar la gestión de portafolio con una apropiada definición de proyectos que considere al menos estas tres fuentes de información:

- Plan estratégico y operativo de la empresa donde se identifiquen los proyectos que van a soportar a este plan.

- Reuniones con distintas áreas, llamadas "reuniones con el cliente interno" para levantar los requerimientos o necesidades de los usuarios de cada área de la empresa.

- Los requerimientos regulatorios y de cumplimiento, como por ejemplo plan de contingencias de los servicios de $\mathrm{Tl}$, proyectos de infraestructura, etc.

Se debe considerar que estos proyectos pueden incrementarse durante la implementación de portafolios.

Una vez identificados los proyectos, se procede a priorizarlos bajo un apropiado balance de costos, riesgos y beneficios. En la publicación [13], indica que hay que tener en cuenta cuatro componentes claves para realizar una evaluación preliminar de los proyectos de $\mathrm{Tl}$, posteriormente se procede a la priorización de los proyectos de $\mathrm{TI}$, estos elementos claves son los siguientes: 1) La evaluación financiera del proyecto, 2) la valoración de los riesgos del proyecto, 3) La valoración estratégica del proyecto y 4) La valoración de la adecuación de los sistemas actuales, la valoración de estos procesos permiten a la TI alinear su estrategia con la estrategia del negocio. Posteriormente es 
necesario establecer un marco de trabajo que permita gestionar todos los proyectos en conjunto.

Monteiro [14] indica que además de funciones como de la definición, planeación, análisis de costo beneficio, administración de riegos, control y monitoreo, entre otras, su principal preocupación se centra en proveer apoyo a los proyectos a través de consultorías, método y estándares, herramientas informáticas, entrenamiento administración recursos para los proyectos (materiales y talento humano).

Según [15] indica que la mejor alternativa es establecer una oficina de gestión de proyectos (OGP) cuya función principal es ser la integradora entre el negocio y los diferentes proyectos de la empresa, consolidando iniciativas individuales en un solo portafolio cuantificable (referente al retorno de la inversión ROI), de fácil seguimiento y sobre todo alineado a la estrategia a largo plazo de la organización.

\section{Metodología de trabajo}

La metodología aplicada en este trabajo es de tipo descriptivo y documental que inicia con la búsqueda y selección bibliográfica por medios electrónicos de artículos que traten el tema referente a la gestión de portafolio de proyectos de TI, metodologías y sus mejores prácticas, por lo que nos planteamos la siguiente pregunta de investigación.

Q1: ¿Cuáles son las mejores prácticas en la gestión del portafolio de proyectos de TI?

Específicamente se trabajó con bases de datos científicas como: ACM, Springer, IEEE con la cadena de búsqueda "IT project portfolio" AND "Best practice" AND "IT project portfolio management" AND "IT methodolgy. Notando que existe información recuperable desde el año 2008. Así mismo se eligió el idioma de las búsquedas el Inglés por ser este el lenguaje de más amplio espectro de trabajo de investigación. La estrategia de selección de artículos fue aplicada a tres niveles, primero en su título, luego en su resumen y finalmente en su contenido, luego de lo cual se realizó la extracción de las mejores prácticas, objetivo del trabajo.

En base a la información recopilada se establece una línea de base referencial de Gestión de Proyectos (GP), Gestión de Portafolio de Proyectos (GPP) y posteriormente la Gestión de Portafolio Proyectos de TI (GPPTI), analizando sus orígenes en la Teoría Moderna de Proyectos (TMP), a continuación se presentan tres metodologías de GPPTI y un conjunto de mejores prácticas (MP) que posibiliten la iniciación laboral de los Administradores de Oficina de Proyectos (AOP) en esta actividad. 


\section{Resultados}

Las metodologías y mejores prácticas que se detallan a continuación son las más representativas y están basadas principalmente en la experiencia de varios autores y empresas que han implementado la gestión de portafolios de proyectos de $\mathrm{Tl}$ en casos prácticos [16], [17], [18].

\subsection{Metodologías de Gestión de Portafolios de Proyectos de TI}

De acuerdo al estudio realizado por [16], [17], [18], las metodologías que recomiendan mejores prácticas son las siguientes:

- Metodología 1: En [17] se presenta una visión gerencial de proyectos con la gestión de portafolios de proyectos de TI en su libro "Running the Succefull Hi Tech Project" en la que considera a la GPPTI como proceso con el objetivo de maximizar los beneficios en consideración del nivel de riesgo de todos los proyectos actuales y futuros. Según la Figura 1 está compuesta de tres fases: fase 1 planificación del Portafolio, la fase 2 de supervisión de proyectos y finalmente la fase 3 , la de control de portafolio de proyectos.

A continuación un breve resumen de cada fase: en la fase 1, los proyectos se alinean con los objetivos del negocio lo que incluye la decisión de qué y cuándo los proyectos se deben ejecutar, considera la predicción de los recursos que serán necesarios así como el flujo de caja necesario, siendo esta fase un proceso dinámico.

La siguiente fase, la de Supervisión de proyectos tiene como objetivo principal tener una idea anticipada del rendimiento individual de cada uno de los proyectos para poder tomar acciones correctivas antes de que afecten todo el portafolio, lo que se logra contando con una línea base y con modelos predictivos basados en datos históricos, la idea general es tener una medida del estado del proyecto haciendo chequeos continuos de su proceso, costo, calidad y motivación de los responsables.

Finalmente la fase 3 , la de Control de portafolio cuyo objetivo es la toma de acciones para compensar o minimizar el impacto de las desviaciones que tiene los proyectos, para ello se revisan cronogramas y la utilización de recursos de los proyectos, las acciones correctivas se las realiza a nivel del plan maestro.

- Metodología 2: Según Brian Cameron [19], "IT Portfolio Management and Mantainening IT Strategic Aligment" es una de las más populares y consta de tres etapas: 1) definición y planificación, 2) implementación y 3) control. A continuación un breve resumen de cada una de ellas. La etapa 1 de definición y planificación, en esta etapa se 


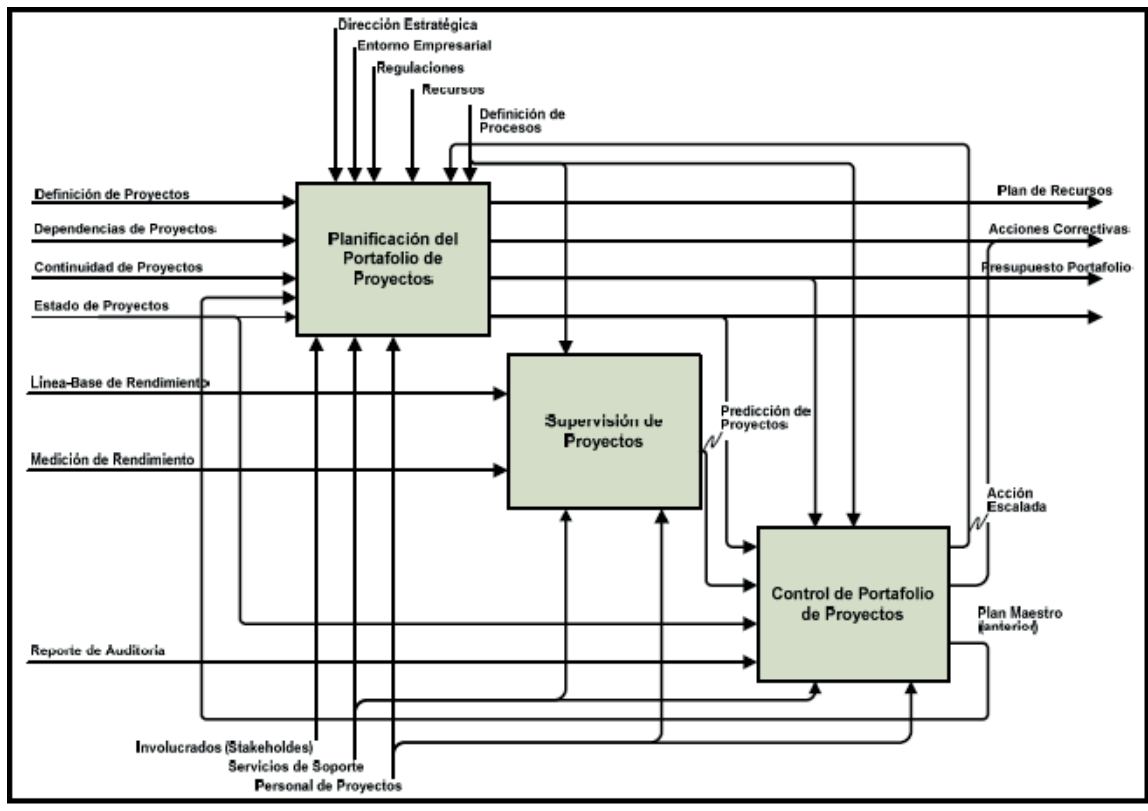

Figura 1: Metodología 1 para la GPPTI [17]

trata de identificar y documentar los componentes clave del portafolio de proyectos, estimar sus costos y beneficios, dicha información es monitoreada centralizadamente de cada proyecto más los gastos e inversión en TI. La etapa 2, la de implementación, estandariza procesos de GPPTI para habilitar una selección objetiva de los proyectos alineados a las estrategias del negocio, reuniones anuales entre el personal de TI y la empresa se realizan y se usan métricas de finanzas para mostrar, categorizar y priorizar proyectos. Finalmente la etapa 3 , la de control, aquí se usan métricas avanzadas para medir los valores generados por el proyecto y así eliminar las iniciativas con bajos rendimientos, también evaluar el riesgo asociado a cada proyecto en términos de retrasos, excesos de costos, poca alineación estratégicas con revisiones constantes del rendimiento entre TI y la empresa.

- Metodología 3: Según Grant et al. [20] que considera la propuesta hecha por Bryan Maizlish y Robert Handler en su libro "IT Portfolio Management, step by step" del año 2005 [12]. Consta de las siguientes etapas: 1) plan/estrategia inicial, 2) planificación, 3) creación, 4) evaluación, 5) balanceo, 6) comunicación, 7) gestión y 8) organización y finalmente 9) evaluación de la ejecución como se muestra en la Figura 2. A continuación un breve resumen de cada una de las etapas: etapa 1, la de plan/estrategia inicial, en esta etapa se define los objetivos del GPPTI, se establecen métricas e indicadores de éxito se documenta el plan que incluye los recursos, actividades, hitos, metas y calendarios, en la etapa 2 la de planificación, se realiza la planificación estratégica de las inversiones, la estructura del portafolio y sub portafolios, a continuación la etapa 3 la de creación, aquí se llena el portafolio, 
identificando riesgos y resultados, conjuntamente con la mejores prácticas, se generan vistas del portafolio para una mejor toma de decisiones.

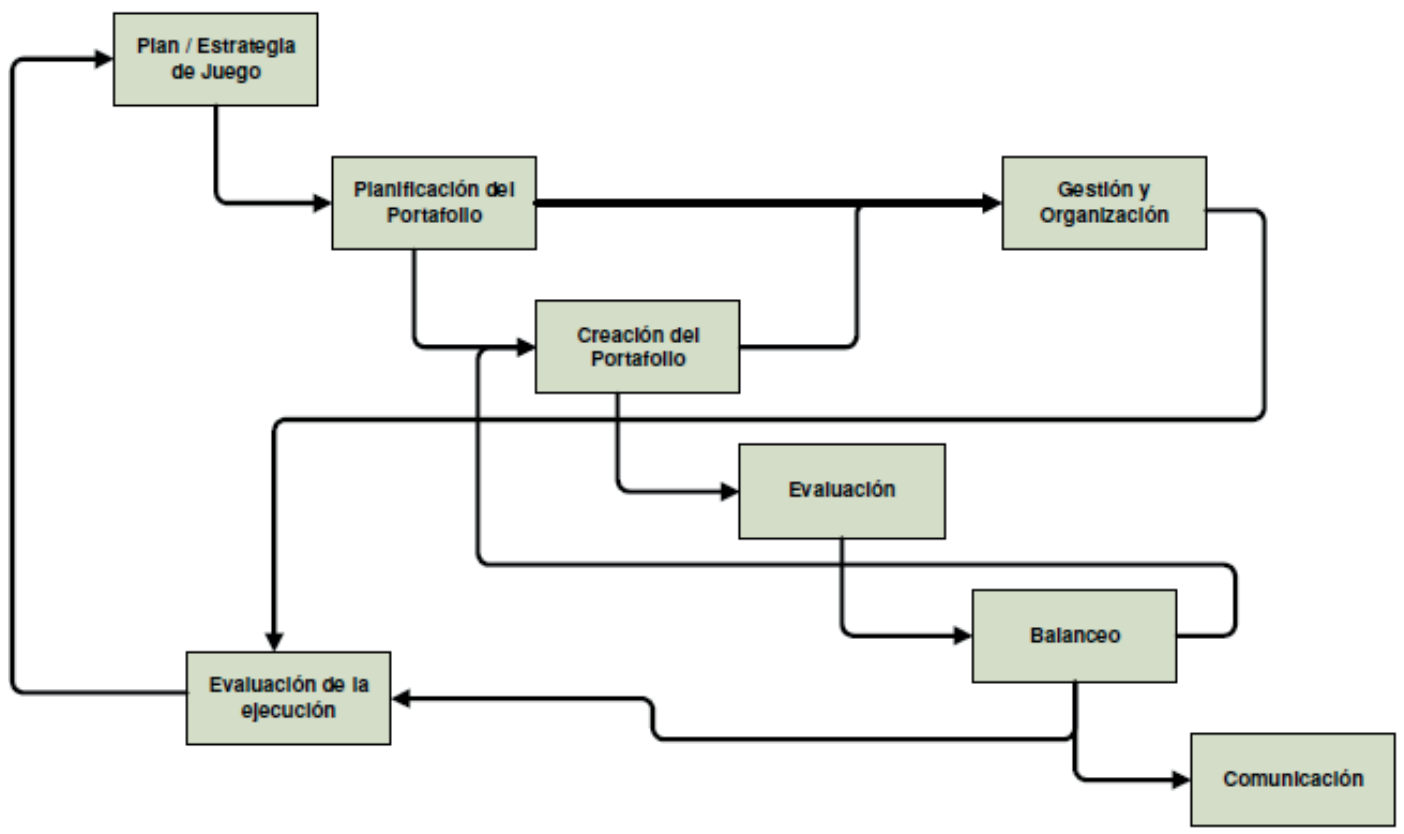

Figura 2: Metodología 3 para la GPPTI. [16].

En la etapa 4, la de evaluación, inicia monitoreando eventos que afecten al portafolio, se mide el portafolio para identificar áreas que deben ser balanceadas. Luego la etapa 5 balanceos, en esta etapa se identifica opciones de ajustes, sus ventajas y desventajas, se aprueban los ajustes y finalmente se implementan los cambios. En la etapa 6 de comunicación, inicia identificando a los interesados, creando paquetes de comunicación y luego se los entrega. En la etapa 7, la de gestión y organización, se establecen políticas de gestión de portafolio, cumplimiento de normas, integración con los procesos de la empresa, se establece una organización y sus procesos de gestión. Finalmente, la etapa de Evaluación de la ejecución, en esta etapa se evalúa la ejecución del plan se realizan comparaciones de la evaluación del rendimiento y se prepara informes de ejecución y evaluación.

Finalmente hay que resaltar que las tres metodologías enfrentan el proceso de la GPPTI con similitudes y diferencias.

\subsection{Mejores Prácticas en la Gestión de Portafolios de Proyectos de TI}

Luego de la revisión de fuentes bibliográficas [7], [9] se identificaron y se agruparon las siguientes 12 mejores prácticas para la GPPTI: 
1. Compromiso con el comité directivo, permite llegar a concesos con el nivel directivo, los objetivos de la empresa.

2. Iniciar la GPPTI desde un enfoque básico, es decir en la aplicación del proceso debe realizarse en fases dado que cada organización es diferente y por lo tanto cuenta con diferentes procesos organizacionales.

3. Alineación con estrategias empresariales, es necesario alinear los procesos GPPTI con los procesos de planificación, muy recomendable utilizar herramientas como el Cuadro de Mando Integral (Balanced Scorecard) [11].

4. Consideración a todos los usuarios (aquellos identificados en la demanda de los proyectos de $\mathrm{TI}$ [2]) involucrados tanto internos como externos, asegurando un espacio de participación abierto a todos.

5. Atención a cambios internos que afecten a la GPPTI, considerar aspectos de índole interno que puedan afectar al GPPTI.

6. Definición clara de metas y objetivos de GPPTI considerando también los riesgos y la manera de minimizarlos.

7. Establecer un proceso formal de GPPTI que permita la identificación, selección, priorización, gestión de cambios y eliminación de proyectos dentro del portafolio de proyectos de TI.

8. Automatización de GPPTI utilizando metodologías y herramientas colaborativas que integren la gestión del portafolio, gestión de proyectos y soporte a la toma de decisiones. Las herramientas deben permitir la centralización de las operaciones de GPPTI desde la planificación hasta la comunicación de resultados.

9. Gestión centralizada mediante un repositorio de proyectos actuales y futuros para lograr una gestión centralizada.

10. Definir métricas para los proyectos que están en el repositorio, es decir adicionalmente a las métricas de cada proyecto es necesario definir métricas comunes que sirvan para comparar entre proyectos como ejemplo, el cálculo de beneficios financieros.

11. Monitoreo y evaluación constante del portafolio y sus proyectos, siempre será vital monitorear los indicadores y métricas de avance y ejecución de los proyectos de tal manera que se cumpla con los resultados esperados. 
12. Finalmente. La comunicación de resultados se debe definir una mecanismo claro para comunicar resultados de manera oportuna al comité directivo así como a todos los involucrados en el portafolio de proyectos de TI, de tal manera que todos comprendan la información presentada y puedan utilizarlas para la toma de decisiones.

En la Tabla 1 se presenta una lista de mejores prácticas que son extraídas de las metodologías analizadas en este trabajo, las mismas que mediante la aplicación de la matriz de Holmes, estrategia que permite identificar el grado de importancia de un conjunto de factores entre sí, comparando la relevancia de cada uno de ellos con el resto de factores, puntuando su importancia con valores que van en el rango de o a 1, o indica no implica ninguna, 1 indica relevancia total de una sobre la otra y un valor de 0,5 que indicaría cualquiera de los dos podría ser; y así determinar los niveles de importancia de las mejores prácticas identificadas, ver la Tabla 2.

Finalmente, en la Tabla 3 se presenta la jerarquización de las mejores prácticas y así poder sugerir mayor atención a las mejores prácticas consideradas como las más relevantes.

TABLA 1: Listado de las Mejores Prácticas de GPPTI.

Listado de Mejores Prácticas para GPPTI
Alineación con las estrategias empresariales
Definición clara de metas y objetivos de la GPPTI
Comunicación de resultados
Gestión centralizada
Monitoreo y evaluación constante del portafolio y sus proyectos
Establecer un proceso formal de GPPTI
Definir métricas comunes para los proyectos dentro del portafolio
Atención a cambios internos que afecten a la GPPTI
Compromiso del comité directivo
Automatización de la GPPTI
Iniciar la GPPTI desde un enfoque básico
Considerar a todos los usuarios involucrados

\section{Análisis de resultados}

Para el análisis de resultados se consideran los dos aspectos relevantes del trabajo, metodologías y mejores prácticas. 
TABla 2: Matriz de Holmes de las Mejores Prácticas de la Gestión de portafolios de proyectos de TI.

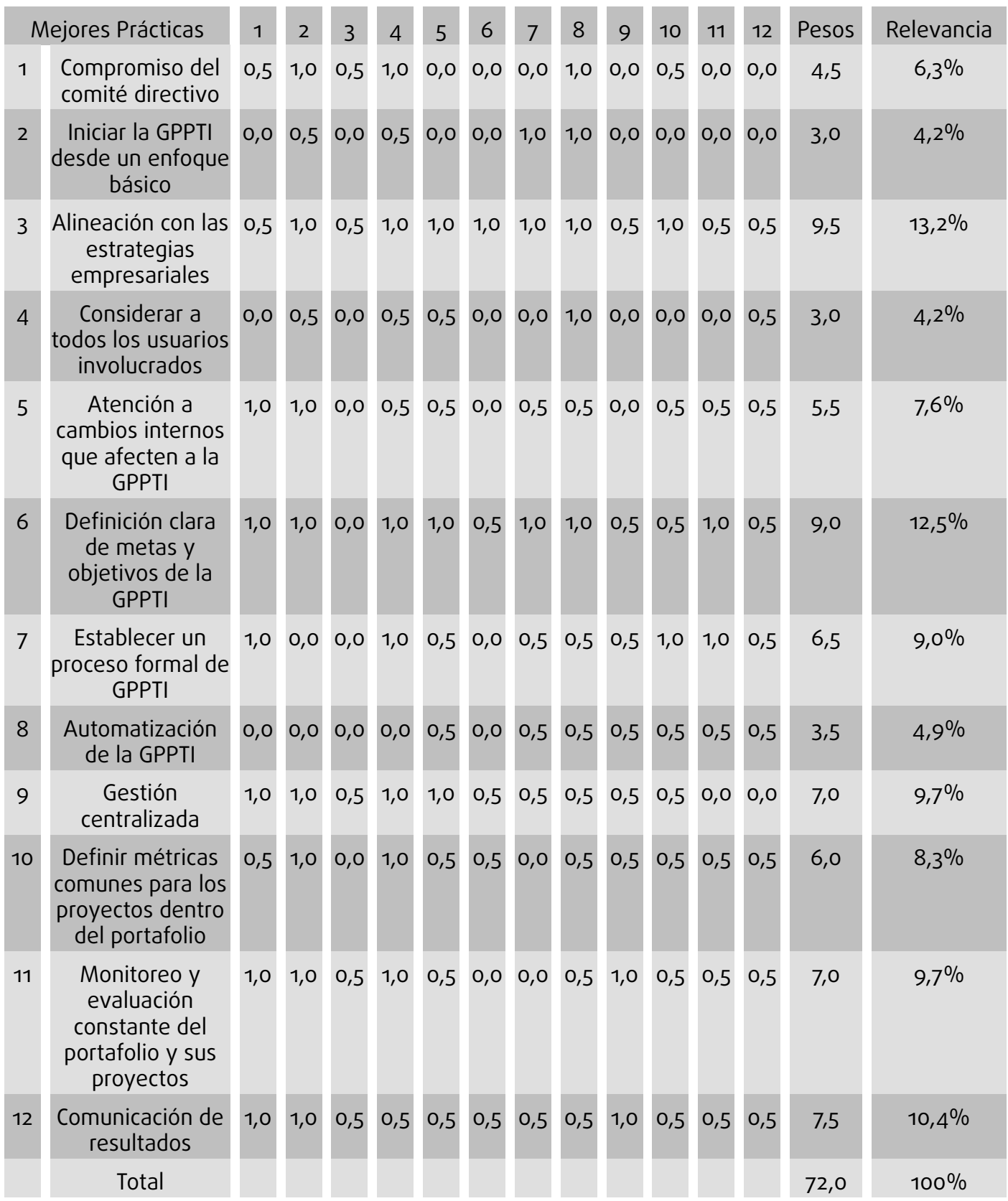

\subsection{Análisis de las metodologías}

Con relación a las metodologías de GPPTI se puede abstraer que ellas enfrentan el proceso de gestión de portafolios de proyectos con similitudes y diferencias ya sean etapas o fases de alguna manera, pero todas ellas realizan 3 etapas, principalmente: planificación, gestión y comunicación, cada una de ellas cuenta con las siguientes fases La etapa Planificación con las siguientes fases: 
TABLA 3: Jerarquización de las Mejores Prácticas de la Gestión de portafolios de proyectos de TI.

\begin{tabular}{|c|c|}
\hline Jerarquización de Mejores Prácticas & \\
\hline Alineación con las estrategias empresariales & $13,2 \%$ \\
\hline Definición clara de metas y objetivos de la GPPTI & $12,5 \%$ \\
\hline Comunicación de resultados & $10,4 \%$ \\
\hline Gestión centralizada & $9,7 \%$ \\
\hline Monitoreo y evaluación constante del portafolio y sus proyectos & $9,7 \%$ \\
\hline Establecer un proceso formal de GPPTI & $9,0 \%$ \\
\hline Atención a cambios internos que afecten a la GPPTI & $8,3 \%$ \\
\hline Compromiso del comité directivo & $7,6 \%$ \\
\hline Automatización de la GPPTI & $6,3 \%$ \\
\hline Iniciar la GPPTI desde un enfoque básico & $4,9 \%$ \\
\hline Considerar a todos los usuarios involucrados & $4,2 \%$ \\
\hline
\end{tabular}

- Análisis situacional de los proyectos

- Identificación de nuevos proyectos

- Eliminación de proyectos duplicados

- Priorización de los proyectos identificados

- Definición del objetivo del portafolio

- Obtención de la aprobación correspondiente por el comité directivo empresarial

Etapa Gestión con las siguientes fases:

- Implementación de una herramienta informática para la GPPTI

- Definición de métricas para evaluar el avance de los proyectos

- Seguimiento del portafolio y proyectos

- Balanceo y gestión de cambios

Etapa Comunicación con las siguientes fases:

- Informar al Comité Directivo sobre acciones correctivas, cambios de portafolio a fin de mantener el estatus de proyectos aprobados.

- Organización periódica de reuniones con diferentes áreas a fin de mantener siempre informados y asegurar el compromiso de los involucrados.

- Descontinuar proyectos que justificadamente no son aporte para los objetivos de la empresa. 


\subsection{Análisis de las mejores prácticas}

Del análisis de las mejores prácticas se obtiene el listado de las12 mejores prácticas, así como la priorización de ellas mediante el uso de la matriz de Holmes, combinado los resultados se puede obtener las etapas más relevantes que todo proceso de GPPTI debe considerar, donde cada etapa cuenta con diferentes fases. De esta manera se da contestación a la pregunta de investigación Q1 planteada al inicio.

La Tabla 2. Muestra los resultados la aplicación de la matriz de priorización de Holmes, obteniéndose en cada fila la relevancia de cada mejor practica versus los demás, así por ejemplo: en la primera fila se tiene a la mejor practica "Compromiso del Comité Directivo" cuya sumatoria de las relevancias es de 4,5, que en consideración de importancia con la suma de todos los pesos $(72,0)$, le corresponde una relevancia de 6,3 de prioridad respecto del resto.

A continuación se presenta el resultado de la jerarquización de las mejores prácticas de GPPTI Tabla 3, de acuerdo a la estrategia anteriormente descrita.

\section{Conclusiones}

En el presente trabajo se ha realizado un análisis de lo que constituye la gestión de Portafolios de Proyectos haciendo énfasis en los proyectos de TI. Iniciando el trabajo con un encuadramiento conceptual de GPPTI con relación a la gestión estratégica de la demanda de proyectos de TI en un ámbito empresarial, posteriormente se presentó conceptos de lo que se maneja hoy en día respecto de la Teoría Moderna de Portafolios desde su visión financiera y de proyectos de TI.

Se analizaron las metodologías más representativas y detalladas para GPPTI conjuntamente con recomendaciones de mejores prácticas que fueron jerarquizadas en una matriz de Holmes, se obtuvo tres etapas básicas que siempre se deben considerar en la GPPTI, ellas son las de planificación, gestión y comunicación, que cuentan con fases a su interior, Además se resalta el hecho de que en su aplicación dependerá del entorno y madurez empresarial.

\section{Referencias}

[1] S. S. Bonham, IT project portfolio management. Artech House, 2005.

[2] I. Aguilar Alonso, J. D. Carrillo Verdún, and E. Tovar Caro, "Importancia de la Gestion del Proceso de la Demanda de TI," Rev. Procesos Metricas RPM, 2008. 
[3] I. Aguilar Alonso, J. Carrillo Verdún, and E. Tovar Caro, "Description of the structure of the IT demand management process framework," Int. J. Inf. Manag., vol. 37, no. 1, pp. 1461-1473, Feb. 2017.

[4] I. A. Alonso, J. C. Verdún, and E. T. Caro, "The Importance of IT Strategic Demand Management in Achieving the Objectives of the Strategic Business Planning," in 2008 International Conference on Computer Science and Software Engineering, 2008, vol. 2, pp. 235-238.

[5] E. Culqui, H. Manolo, G. González, and J. Fernando, "Mejoras prácticas para la gestión de portafolio de proyectos de IT-aplicación a un laboratorio farmacéutico," 2009.

[6] J. B. Williams, The Theory of Investment Value, Reprint edition. Fraser Publishing Co., 1997.

[7] H. Markowitz, "Portfolio Selection*," J. Finance, vol. 7, no. 1, pp. 77-91, Mar. 1952.

[8] A. O. Petters and X. Dong, "Markowitz Portfolio Theory," in An Introduction to Mathematical Finance with Applications, New York, NY: Springer New York, 2016, pp. 83-150.

[9] I. Aguilar Alonso, J. D. Carrillo Verdún, and E. Tovar Caro, "Importancia de la Gestion del Proceso de la Demanda de TI," Rev. Procesos Metricas RPM, vol. 5, no. 2, Pp. 25-34, May 2008.

[10] "PMI | Project Management Institute." [Online]. Available: https://www.pmi.org/. [Accessed: 27-Jul-2017].

[11] R. Xue, C. Baron, P. Esteban, and L. Zheng, "Integrating the ISO/IEC 15288 Systems Engineering Standard with the PMBoK Project Management Guide to Optimize the Management of Engineering Projects," in Complex Systems Design \& Management, Springer, Cham, 2016, pp. 291-291.

[12] B. Maizlish and R. Handler, It Portfolio Management Step-by-step: Unlocking the Business Value of Technology. Chichester, UK: John Wiley and Sons Ltd., 2005.

[13] I. A. Alonso, J. C. Verdún, and E. T. Caro, "Project Prioritization as a Key Element in IT Strategic Demand Management," in Innovations and Advances in Computer Sciences and Engineering, T. Sobh, Ed. Dordrecht: Springer Netherlands, 2010, pp. 417-422.

[14] A. J. V. Monteiro, "Project management office (PMO)?: typologies and models," Jan. 2017.

[15] C. Bredillet, S. Tywoniak, and M. Tootoonchy, "Exploring the dynamics of project management office and portfolio management co-evolution: A routine lens," Int. J. Proj. Manag., May 2017. 
[16] "Monteforte: Doing IT Right. 8 Steps to Project Portfolio... - Google Académico." [Online]. Available: https://scholar.google.es/scholar?cluster = 12046626005537922228hl=es\&as_sdt=2005\&sciodt=0,5. [Accessed:12-Jul-2017].

[17] E. Miranda, Running the Successful Hi-tech Project Office. Artech House, 2003.

[18] C. Verhoef, "Quantitative IT portfolio management," Sci. Comput. Program., vol. 45, no. 1, Pp. 1-96, Oct. 2002.

[19] B. H. Cameron, "IT Portfolio Management: Implementing and Maintaining IT Strategic Alignment," Httpservicesigi-Glob.-1-59140-908-3choo3, Pp. 38-67, 2006.

[20] G. G. Grant and R. Collins, "IT Investment Portfolio," in The Value Imperative, Palgrave Macmillan, New York, 2016, pp. 113-123.

[21] Project Portfolio Management..

[22] Y.-C. Shen, P.-S. Chen, and C.-H. Wang, "A study of enterprise resource planning (ERP) system performance measurement using the quantitative balanced scorecard approach," Comput. Ind., vol. 75, pp. 127-139, Jan. 2016. 